\title{
Implementación de telerradiología en neurotrauma en áreas de difícil acceso -revisión sistemática
}

Loraine De Jesús Quintana-Pájaro ${ }^{1}$ (D), Yelson Alejandro Picón-Jaimes² (D), Yancarlos Ramos-Villegas ${ }^{1}$ (D), Aylin Daguer-Menco ${ }^{1}$ (D), Yandris Arévalo-Martínez ${ }^{1}$ (D), Eileen Cortecero-Sabalza ${ }^{1}$ (D), Javier Esteban Orozco Chinome ${ }^{3}$ (D), Luis Rafael Moscote-Salazar ${ }^{4}$ (D)

\section{RESUMEN}

Introducción: Según la organización mundial de la salud los traumatismos representan más del 20\% de los problemas en salud en el mundo. El trauma craneoencefálico y la ausencia de servicios de neurocirugía y radiología en zonas menos pobladas dificultan la valoración y manejo de pacientes con lesión cerebral. Objetivo: Describir los hallazgos clínicos y beneficios derivados de la implementación de la telerradiología en neurotrauma en áreas de difícil acceso geográfico. Materiales y métodos: Se realizó una búsqueda sistemática en Pubmed, Scopus, Ebsco host, Sciencedirect, y Embase, con los tesauros "Teleradiology" y "Craniocerebral Trauma". Resultados: La decisión de intervenir a un paciente con traumatismo cerebral y el periodo de tiempo hasta la cirugía son fundamentales para el desenlace clínico. Aquellos centros que usan la telerradiología, precisan los traslados a los hospitales especializados, por lo cual los dispositivos tecnológicos portátiles contribuyen en el tiempo de respuesta de la atención en neurocirugía. Conclusión: La telerradiología impacta positivamente en pacientes con trauma craneoencefálico en zonas geográficas de difícil acceso, facilitando la comunicación con especialistas; brindando atención oportuna y optimizando los traslados a centros de alta complejidad.

Palabras clave: Traumatismos craneocerebrales; Radiología; Telerradiología; Telemedicina; Traumatismo craneoencefálico.

\section{INTRODUCCIÓN}

Las emergencias neuroquirúrgicas en los servicios de urgencias representan cifras significativamente altas y en casos de situaciones de riesgo vital requieren de diagnósticos rápidos y precisos que permitan reducir la mortalidad de los pacientes. La telerradiología (TR) ha revolucionado la velocidad del diagnóstico con la consiguiente implementación de terapias que llevan a un adecuado tratamiento en tiempos más cortos desde el ingreso de los pacientes (1).

La TR, consiste en un sistema de transferencia de imágenes radiológicas por medio de computadoras que ha estado presente desde la década de los 80 's, pero su existencia es tal vez mucho más antigua y data desde que los radi- ólogos hicieron su aparición como especialistas (2). Inicialmente, las imágenes simples eran tomadas en una institución y enviadas posteriormente a radiólogos para su interpretación; actualmente, con las mejores coberturas de internet, la implementación de los sistemas de archivo y comunicación de imágenes (PACs) y la prestación a cualquier hora de este servicio, especialmente durante la jornada nocturna, se ha dado apertura a un servicio más digitalizado y dinámico (3).

Entre los usos más frecuentemente descritos de este sistema figuran la prestación de servicios de consulta médica desde cualquier sitio, la comunicación entre estudiantes practicantes con especialistas de otros lugares geográficos y la determinación de transferir o no a un paciente a un centro de mayor complejidad o la necesidad de

\footnotetext{
1. Médico Universidad de Cartagena, Cartagena de Indias, Colombia

2. Médico cirujano, Máster en Bioética, Docente Universidad Pedro de Valdivia, Santiago, Chile

3. Médico cirujano, Red Salud, Santiago, Chile

4. Neurocirujano. Facultad de Medicina - Universidad de Cartagena. Cartagena de Indias, Bolívar
} 
un procedimiento quirúrgico de manera inmediata guiado a través de esta tecnología (4).

Un estudio publicado en el British Journal of Neurosurgery señaló como la implementación del servicio de telerradiología en una comunidad hospitalaria China que recibía pacientes de áreas geográficas apartadas, redujo las transferencias innecesarias de pacientes en un $21 \%$, disminuyendo también los eventos adversos durante la transferencia y el tiempo total de transferencia de los pacientes, lo cual resultaba en una mejora en el pronóstico de los enfermos (2).

Por tanto, este servicio de comunicación remoto con profesionales que se encuentran en centros de atención de alta complejidad hospitalaria favorece y apoya la labor de aquellos profesionales de salud que se encuentran en zonas de difícil acceso como lo pueden ser los centros médicos rurales apartados o que no cuentan con los insumos necesarios para brindar la atención en salud requerida en ciertos escenarios como el neurotrauma (4).

Teniendo en cuenta que la Organización mundial de la salud considera que los traumatismos constituyen una epidemia desatendida, y entre ellos los que más mortalidad generan son los traumatismos craneoencefálicos, especialmente en países en vía de desarrollo (5). Se consideró importante llevar a cabo este trabajo de investigación, que abordó la temática y desveló soluciones al respecto para dar impacto mediante la apropiación del conocimiento recopilado.

\section{OBJETIVO}

Para desarrollar la revisión se siguieron los pasos de la estrategia PICO-SD (Patients, intervention, comparation, outcomes, study design). La pregunta de investigación establecida fue: ¿Es la telerradiología una intervención que impacta positivamente en el desenlace de los pacientes con neurotrauma en cuanto a disminución en las tasas de morbi-mortalidad en lugares geográficamente apartados?

\section{MATERIALES Y MÉTODOS}

\section{Criterios de inclusión}

Tipos de participantes: Esta revisión involucró los estudios realizados en individuos mayores de 18 años con injuria cerebral traumática. Se tuvieron en cuenta estudios realizados desde 1997 hasta el 2020 escritos en idioma español, inglés o portugués.

Concepto: La revisión abarcó los estudios que implementaron la telerradiología como medio de comunicación para tomar una conducta diagnóstica y terapéutica. Se determinó como resultado primario la disminución en las tasas de morbi-mortalidad secundario a trauma. Los resultados secundarios incluyeron: reducción en la tasa de transporte innecesario a unidades de mayor complejidad, reducción de costos y del tiempo e interpretación de las imágenes.

Contexto: Los estudios incluidos abordaron la instauración del sistema de telerradiología en áreas rurales y/o que no contaban con equipo de neurocirujanos y neurorradiólogos. Lo anterior, permitió conocer el impacto de esta implementación.

Tipos de estudios: Se tuvo en cuenta los estudios observacionales, ensayos clínicos controlados aleatorios y no aleatorios, diseños cuasi experimentales, estudios de cohorte de carácter prospectivo o retrospectivo y estudios casos y controles. No se tuvo en cuenta publicaciones informales, pósteres o literatura gris.

\section{Estrategia de búsqueda y selección de estudios}

Esta revisión siguió las recomendaciones de la metodología propuesta por Joanna Briggs Institute. Además, se utilizó el flujograma PRISMA elaborado por la Colaboración Cochrane. Se realizó una búsqueda bibliográfica en las bases de datos: Pubmed, Embase, Ebsco Host, Scopus, Sciencedirect, considerando todas las publicaciones realizadas desde 1997 hasta el 2020. La búsqueda se efectuó en 5 pasos: primero se identificaron 
las palabras claves utilizando el MesH (Medical Subject Headings) y DeCs (Descriptores de la Salud), luego se procedió a utilizar los descriptores hallados (Teleradiology y Craniocerebral trauma) en las bases de datos, obteniendo en Pubmed 26 artículos, en Sciencedirect 15, en Ebsco Host 7, en Embase 40 y en Scopus 15. Asimismo, se eliminaron los artículos duplicados (paso 2) quedando un total de 67 manuscritos y se procedió con la evaluación de títulos y resúmenes arrojados (paso 3); quedando de este modo 20 artículos. Luego se realizó la revisión a texto completo de los artículos seleccionados (paso 4), obteniendo 17 textos y finalmente se revisaron las referencias de los artículos incluidos para identificar aquellos estudios que también cumplían con los criterios de elegibilidad (paso 5); adicionando 4 manuscritos. En total fueron 21 manuscritos los utilizados en este trabajo.

\section{Método de revisión}

La estrategia de búsqueda y los estudios seleccionados fueron evaluados por dos revisores independientes. Las discrepancias fueron discutidas con un tercer revisor y resueltas por consenso. La presente revisión no cuenta con un protocolo registrado en el National Institute for Health Research ni en PROSPERO.

\section{Recopilación de datos}

Los siguientes datos se extrajeron de los estudios que cumplían los criterios de elegibilidad: autores, año de publicación, número de par- ticipantes, tipo de estudio, objetivo del estudio, resultados del estudio, medio de telerradiología usado, costos de la telerradiología, transmisión e interpretación de imágenes, examen imagenológico enviado por telerradiología y diagnósticos reportados, discrepancias en el diagnóstico y fallas del sistema. Estos datos fueron recopilados en una tabla de datos en Microsoft Excel.

\section{Síntesis de datos y análisis adicionales}

Con el programa SPSS (Chicago, II) versión 25 se determinó la frecuencia absoluta y relativa de la variable: Medio usado para telerradiología.

La calidad y el riesgo de sesgo de los manuscritos fue evaluado utilizando herramientas para la lectura crítica de artículos; de este modo para los observacionales se utilizó la herramienta STROBE, para las series de casos OSTEBA y para los ensayos clínicos CASPE. Se determinó como punto de corte una aprobación mayor al $70 \%$ de los ítems de las diferentes herramientas de lectura crítica.

\section{RESULTADOS}

\section{Selección de estudios}

El proceso de selección de los estudios se basó en los fundamentos PRISMA como se observa en la figura 1 . Se consideraron 21 artículos de los 103 arrojados por la búsqueda bibliográfica. En la tabla 1 y 2 se recogen la descripción y principales características de los estudios incluidos. 
Figura 1. Proceso de selección de los artículos.
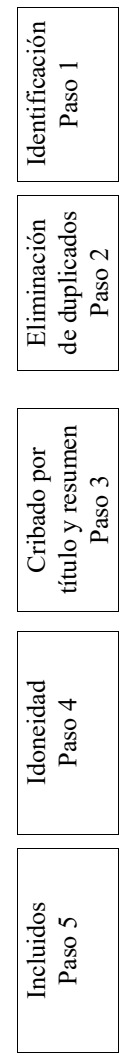

Número de artículos identificados mediante búsqueda en bases de datos $(n=103)$ : Pubmed $(n=26)$, Sciencedirect $(n=15)$, EbscoHost $(n=7)$, Embase $(n=40)$, Scopus $(n=15)$. (n=15), EbscoHost $(\mathrm{n}=7)$, Emb
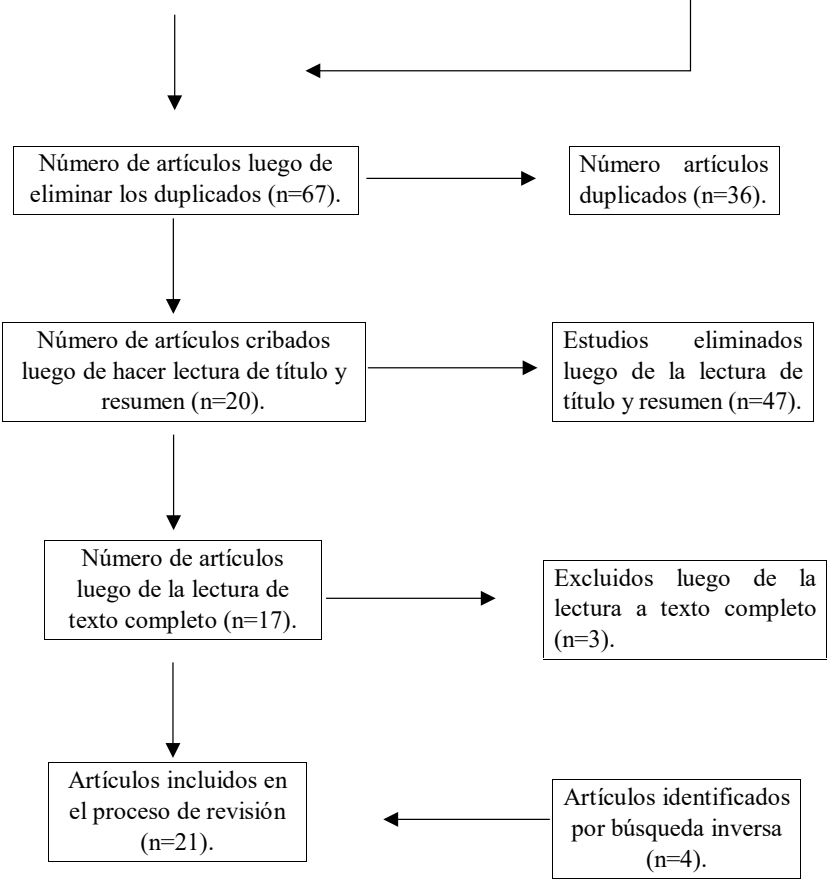
Tabla 1. Características generales de los artículos incluidos $(\mathbf{n = 2 1 )}$

\begin{tabular}{|c|c|c|}
\hline Características & Número & Porcentaje (\%) \\
\hline $\begin{array}{l}\text { Año de publicación } \\
<2000 \\
2000-2004 \\
2005-2009 \\
2010-2014 \\
2014-2018\end{array}$ & $\begin{array}{l}2 \\
7 \\
6 \\
1 \\
5\end{array}$ & $\begin{array}{l}9,52 \% \\
33,33 \% \\
28,57 \% \\
4,76 \% \\
23,81 \%\end{array}$ \\
\hline $\begin{array}{l}\text { Número de pacientes } \\
\text { Estudios que especifican el género } \\
\text { Hombres } \\
\text { Mujeres }\end{array}$ & $\begin{array}{l}27867 \\
9(16489) \\
8827 \\
7025\end{array}$ & $\begin{array}{l}53,53 \%(\text { De n: } 16489) \\
42,60 \%\end{array}$ \\
\hline $\begin{array}{l}\text { Estudios que reportan costos de la te } \\
\text { Sí } \\
\text { No }\end{array}$ & $\begin{array}{l}4 \\
17\end{array}$ & $\begin{array}{l}19,05 \% \\
80,95 \%\end{array}$ \\
\hline $\begin{array}{l}\text { Estudios que reportan fallas o discrep } \\
\text { Si } \\
\text { No }\end{array}$ & $\begin{array}{l}6 \\
15\end{array}$ & $\begin{array}{l}28,57 \% \\
71,43 \%\end{array}$ \\
\hline $\begin{array}{l}\text { Estudios que reportan reducción del t } \\
\text { Sí } \\
\text { No }\end{array}$ & $\begin{array}{l}3 \\
18\end{array}$ & $\begin{array}{l}14,29 \% \\
85,71 \%\end{array}$ \\
\hline $\begin{array}{l}\text { Estudios que evalúan el tiempo de tra } \\
\text { sistema } \\
\text { Sí } \\
\text { No }\end{array}$ & $\begin{array}{l}4 \\
17\end{array}$ & $\begin{array}{l}19,05 \% \\
80,95 \%\end{array}$ \\
\hline
\end{tabular}


Tabla 2. Resultados de los estudios

\begin{tabular}{|c|c|c|c|c|c|}
\hline Autores & País & Año & $\begin{array}{l}\text { No de } \\
\text { partici- } \\
\text { pantes }\end{array}$ & $\begin{array}{l}\text { Tiempo del estudio y } \\
\text { tipo de estudio }\end{array}$ & Resultados \\
\hline $\begin{array}{l}\text { Alías et } \\
\text { al(11) }\end{array}$ & Malasia & 2013 & 372 & $\begin{array}{l}\text { Diciembre } \\
2009 \text { a Julio } 2010 \\
\text { Serie de casos }\end{array}$ & $\begin{array}{l}\text { Reducción en un } 66 \% \text { del transporte innece- } \\
\text { sario, mejoría en la precisión diagnóstica }\end{array}$ \\
\hline $\begin{array}{l}\text { Reponen } \\
\text { et al (13) }\end{array}$ & Finlandia & 2000 & 21 & $\begin{array}{l}\text { No específica } \\
\text { Observacional }\end{array}$ & $\begin{array}{l}\text { El neurorradiólogo consideró que la consulta } \\
\text { de imagen evitó la visita a un hospital más } \\
\text { especializado en el } 71 \% \text { de los casos. }\end{array}$ \\
\hline $\begin{array}{l}\text { Goh et al } \\
(17)\end{array}$ & $\begin{array}{l}\text { Hong } \\
\text { Kong, } \\
\text { China }\end{array}$ & 1997 & 63 & $\begin{array}{l}\text { Marzo de } 1995 \text { a mayo } \\
1996 \\
\text { Observacional }\end{array}$ & $\begin{array}{l}\text { Menor incidencia de eventos secundarios } \\
\text { al traslado del paciente en el grupo guiado } \\
\text { por telerradiología frente a los referidos sin } \\
\text { esta ayuda }(6,4 \% \text { vs } 32 \%) \text {, asimismo se } \\
\text { encontraron más intervenciones en el primer } \\
\text { grupo en comparación al segundo ( } 6.4 \% \text { vs. } \\
32.1 \%, p=0.01) \text {. }\end{array}$ \\
\hline $\begin{array}{l}\text { Goh et al } \\
(2)\end{array}$ & $\begin{array}{l}\text { Hong } \\
\text { Kong, } \\
\text { China }\end{array}$ & 1997 & 116 & $\begin{array}{l}\text { Marzo a diciembre del } \\
1996 \\
\text { Observacional }\end{array}$ & $\begin{array}{l}\text { Se encontró una reducción en el número de } \\
\text { transferencia innecesarias }(21 \%) \text {, al igual } \\
\text { que una menor tasa de eventos secundarios } \\
\text { al traslado ( } 8 \% \text { vs } 32 \% \text { ) en el grupo con } \\
\text { implementación de la telerradiología. }\end{array}$ \\
\hline $\begin{array}{l}\text { Latifi et } \\
\text { al(29) }\end{array}$ & Albania & 2018 & 146 & $\begin{array}{l}2014-2016 \\
\text { Observacional }\end{array}$ & $\begin{array}{l}\text { El } 66 \% \text { de los pacientes no necesitaron una } \\
\text { remisión a un centro de mayor nivel. De } \\
\text { los pacientes remitidos el } 6 \% \text { fallecieron. } \\
\text { Respecto al modelo de telerradiología, la } \\
\text { tecnología asincrónica (almacenamiento y } \\
\text { retransmisión) representó el ( } 84 \% \text { ), mien- } \\
\text { tras que la consulta en vivo más un plus de } \\
\text { video ya grabado representó el } 15 \% \text {. }\end{array}$ \\
\hline $\begin{array}{l}\text { Pagani et } \\
\text { al (15) }\end{array}$ & Finlandia & 2003 & 30 & $\begin{array}{l}6 \text { meses } \\
\text { Observacional }\end{array}$ & $\begin{array}{l}\text { Los diagnósticos fueron correctos en } 82 \text { de } \\
\text { las } 90 \text { interpretaciones ( } 91 \% \text { ) y las imáge- } \\
\text { nes fueron suficientes para el diagnóstico } \\
\text { final en el } 90 \% \text { de los casos. }\end{array}$ \\
\hline $\begin{array}{l}\text { Erly, et al } \\
\text { (7) }\end{array}$ & $\begin{array}{l}\text { Estados } \\
\text { Unidos }\end{array}$ & 2003 & 716 & $\begin{array}{l}\text { Julio } 2000 \text { a marzo } 2001 \\
\text { Observacional }\end{array}$ & $\begin{array}{l}\text { Se encontró un acuerdo entre la interpretaci- } \\
\text { ón inicial del radiólogo general y la interpre- } \\
\text { tación final del neurorradiólogo en el } 95 \% \text { de } \\
\text { las tomografías }\end{array}$ \\
\hline $\begin{array}{l}\text { Latifi et al } \\
(26)\end{array}$ & Albania & 2016 & 974 & $\begin{array}{l}\text { Enero } 2014 \text { a agosto de } \\
2015 \\
\text { Observacional }\end{array}$ & $\begin{array}{l}\text { De } 974 \text { teleconsultas, } 157 \text { correspondieron } \\
\text { a neurotrauma. El } 63 \% \text { de estos casos no } \\
\text { ameritó la transferencia. Los neurocirujanos } \\
\text { representaron el } 16 \% \text { del equipo requerido } \\
\text { para revisar las teleconsultas. La tecnología } \\
\text { asíncrona representó casi dos tercios de } \\
\text { todas las teleconsultas }(63.7 \%) \text {. }\end{array}$ \\
\hline $\begin{array}{l}\text { Yamada et } \\
\text { al (14) }\end{array}$ & Japón & 2003 & 127 & $\begin{array}{l}2 \text { años } \\
\text { Observacional }\end{array}$ & $\begin{array}{l}\text { El tiempo promedio trascurrido entre el } \\
\text { envío y recepción de las neuroimágenes fue } \\
\text { de } 2-3 \text { minutos. El sistema de transferencia } \\
\text { de imágenes fue útil en todos los casos para } \\
\text { un diagnóstico correcto y un tratamiento } \\
\text { temprano. }\end{array}$ \\
\hline
\end{tabular}




\begin{tabular}{lllll}
\hline Autores País Año & $\begin{array}{l}\text { No de } \\
\text { partici- } \\
\text { pantes }\end{array}$ & $\begin{array}{l}\text { Tiempo del estudio y } \\
\text { tipo de estudio }\end{array}$ & Resultados \\
& &
\end{tabular}

\begin{tabular}{lllll}
\hline Franczak & Estados & 2014 & 2000 & $\begin{array}{l}\text { Octubre a diciembre de } \\
\text { et al (23) } \\
\text { Unidos }\end{array}$ \\
& & & Observacional
\end{tabular}

\section{Zulu et al Sudá- \\ (6) frica}

Servadei
et al (27)

Italia
$2002 \quad 1099$

Wong, Hoi-tung,

Hong

et al (10) China

Ashkenazi Israel $2015 \quad 819$

et al (9)

$\begin{array}{rlr}2007 & 86 \text { pa- } & \text { julio 1997-dic 1998/ } \\ \text { cientes } & \text { enero -junio 2001 } \\ \text { (estudio } & \text { Observacional } \\ \text { piloto), } & \\ & \text { 230 } & \\ \text { pacientes } & \\ & \text { (estudio } & \\ & \text { principal) }\end{array}$

enero 1997- diciembre

2000

Octubre 1998- septiembre 2001

Intervención aleatorizada

1 de enero de 2006 al 31 de diciembre 2011 Serie de casos

1 de enero, 2010 al 31 de mayo de 2013. Observacional

Observacional

Mrak et al blica de

(16) Croacia

Kreutzer

Alemania

1995- 2000
Observacional

1998- 2006

radiólogos acordaron que la información derivada de los registros electrónicos de datos del paciente más la telerradiología pueden influenciar en la interpretación de imágenes y conducta terapéutica

El 84\% pacientes fueron manejados en la GSU y $51(16 \%)$ requirieron transferencia a una unidad neuroquirúrgica. La tasa de mortalidad fue del $13 \%$. El puntaje de la escala de Glasgow $>8$ fue un factor pronostico significativo $(p<0,0001)$

De 1099 pacientes ingresados por injuria traumática, a 637 se le realizaron exámenes iniciales y de estos sólo el $23 \%$ requirieron trasferencia a unidades de neurocirugía y de los 206 con exámenes de seguimiento, sólo el (10) $5 \%$ ameritaron un traslado. Por ende, el sistema redujo las trasferencias innecesarias.

De los 3 modos de consulta (TLR, VC y CF), la telerradiología mostró mayor precisión diagnostica (89.1, 87.7\%, 63.8\%; $P<0.001$ respectivamente). Asimismo, la TLR mostró una tasa de resultados favorables del $61 \%$ frente al $54 \%$ de la VC y $54 \%$ del CF.

EI ISS en los grupos de transferencias tempranas y tardías fue más alto que en aquellos inicialmente hospitalizados en HYMC y no transferidos más tarde ( $p<0,0001$. AIS fue mayor en pacientes transferidos (inicialmente o más tarde, $\mathrm{p}<0.0001$ ). Solo 23 resultaron ser fallas reales en la intención de tratar

La apreciación general de los clínicos fue positiva $(4.3 / 5)$. El tiempo de intervención se redujo y se optimizaron los recursos.

Del total de pacientes, sólo el $31 \%$ de los pacientes fueron remitidos a un centro de trauma de nivel I. Además, de los pacientes en los que se decidió sólo la observación, en el $4 \%$ se presentaron fallas en la intención de tratar.

Observacional
De los 1024 pacientes, 280 correspondían a trauma y de estos sólo el $41 \%$ fueron ingresados al departamento de neurocirugía mediante las decisiones tomadas por TLR. Por otro lado, las imágenes enviadas por TLR están lista para su lectura luego de 5-8 min, lo que permitió tomar una conducta diagnostica más rápida. 


\begin{tabular}{|c|c|c|c|c|c|}
\hline Autores & País & Año & $\begin{array}{l}\text { No de } \\
\text { partici- } \\
\text { pantes }\end{array}$ & $\begin{array}{l}\text { Tiempo del estudio y } \\
\text { tipo de estudio }\end{array}$ & Resultados \\
\hline $\begin{array}{l}\text { Ashkenazi } \\
\text { et al (22) }\end{array}$ & Israel & 2007 & 209 & $\begin{array}{l}1 \text { de agosto de } 2003 \text { - } \\
31 \text { de agosto de } 2005 \\
\text { Observacional }\end{array}$ & $\begin{array}{l}\text { De las } 209 \text { víctimas de trauma con patología } \\
\text { neuroquirúrgica que necesitan hospitalizaci- } \\
\text { ón, } 126(60,2 \%) \text { fueron transferido inme- } \\
\text { diatamente mientras que } 83(39,7 \%) \text { de los } \\
\text { pacientes fueron hospitalizados en el centro } \\
\text { de trauma de nivel } 2 \text { rural para la observa- } \\
\text { ción. Con } 2.4 \% \text { de fallas en la intención de } \\
\text { tratar localmente. }\end{array}$ \\
\hline $\begin{array}{l}\text { Yaghmai et } \\
\text { al (19) }\end{array}$ & $\begin{array}{l}\text { Estados } \\
\text { Unidos }\end{array}$ & 2004 & 1 & Observacional & $\begin{array}{l}\text { Se transmitió una TC de cabeza completa } \\
\text { al PDA inalámbrico de un radiólogo para su } \\
\text { consulta. Todo el procedimiento (incluida la } \\
\text { captura, transmisión y revisión de imágenes) } \\
\text { tomo aproximadamente } 115 \text { minutos. }\end{array}$ \\
\hline $\begin{array}{l}\text { Fabbri et } \\
\text { al (20) }\end{array}$ & Italia & 2008 & 865 & $\begin{array}{l}\text { No específica } \\
\text { Observacional }\end{array}$ & $\begin{array}{l}\text { Entre los dos modelos evaluados (Obser- } \\
\text { vación en hospital periférico más repetición } \\
\text { de TAC y consulta por TLR; Seguimiento en } \\
\text { NSU) no hubo diferencias significativas con } \\
\text { relación al pronóstico de los pacientes. }\end{array}$ \\
\hline $\begin{array}{l}\text { Poca et al } \\
\text { (21) }\end{array}$ & España & 2004 & 165 & $\begin{array}{l}\text { 1997-2002 } \\
\text { Observacional }\end{array}$ & $\begin{array}{l}\text { Se incrementó el número de TAC en los pa- } \\
\text { cientes que habían sufrido un TCE, además } \\
\text { se evitaron traslados innecesarios a los hos- } \\
\text { pitales de III nivel y se menciona una mayor } \\
\text { agilización de la labor asistencial en el área } \\
\text { de urgencias }\end{array}$ \\
\hline
\end{tabular}

TLR (Telerradiología), VC (Vídeo-consulta), CT (Consulta por teléfono ) GSU (Unidad de cirugía general), NSU (Unidad de neurocirugía) ISS (Score de severidad de injuria) HYMC (Hillel Yaffe Medical Center)

\section{Instrumentos usados para hacer la telerra- diología}

Los medios de TR que usaron los estudios incluidos fueron: teléfono y otro medio, PDA (sistema que combina teléfono y mensajería), aplicaciones, vídeos (pregrabados o en vivo), consultas en línea, Web Pad, PACS (sistema de almacenamiento, recuperación y comunicación) y EHR (registros electrónicos de salud).

De los estudios incluidos, 4 en su metodología, no especificaron el medio que usaron para llevar a cabo la telerradiología (6-9). El uso de teléfono, acompañado de otro medio como (sistema de referencia de imágenes análogas, computadora, modem, fax, comunicación digital, mensajería multimedia) fue el medio más usado por los autores de los estudios (43\%) en comparación con el uso de otros medios diferentes a la aplicación y PDA que constituyeron un $20 \%$. Por lo que se evidenció que de cada 100 estudios que emplean un instrumento específico para realizar la telerradiología, 43 optan por líneas telefónicas.

Respecto a los estudios que utilizaron el sistema PDA, también usaron como medio alterno el e-mail y la línea telefónica, solo que este sistema fue el principalmente usado para la telerradiología.

\section{Reducción del transporte innecesario y tiempo}

Se identificaron 3 estudios que evaluaron la reducción del transporte innecesario. Wong et. Al, no observaron diferencias en el número de transferencias innecesarias entre los tres modos de consulta evaluados (consulta por teléfono, por telerradiología y videoconferencia), encontrando que el $11.4 \%$ de los pacientes transferidos podrían haber sido manejados sin transferencia y la precisión diagnóstica del modo de consulta por teléfono $(63,8 \%)$ fue significativamente menor que 
la de los modos de atención por telerradiología $(89,1 \% ; \mathrm{P}<0,0005)$ y videoconferencia $(87,7 \%$; $\mathrm{P}<0,0005)$ (10). Luego, Alias et. Al, reportaron una reducción del $66 \%$ de transportes innecesarios secundario a la mejoría en la precisión diagnóstica, especialmente cuando las imágenes eran interpretadas por médicos radiólogos (11), además Darío et. Al, reportaron que la transferencia de pacientes que necesitan asesoramiento neuroquirúrgico se reducían gracias a la telemedicina y especialmente a la consulta con especialistas de centros médicos de mayor complejidad (12).

La evaluación del tiempo de transmisión y respuesta fue evaluada en 4 estudios, Reponen et. Al y Yamada et. Al, reportaron un tiempo medio desde la captación hasta la recepción en la central, de 90 segundos y de 120 a 180 segundos respectivamente $(13,14)$. Pagani et. Al, encontraron que el tiempo total de trabajo por diagnóstico fue de 5 min con 25 segundos con un rango de 2-12 min el cual comprendía la lectura de la historia clínica, observación de las imágenes en miniatura, revisión de las imágenes originales y llenado del cuestionario de reporte (15). Darío et. Al, mencionaron que el sistema de teleconferencia tenía una tasa de respuesta por parte de la entidad consultada del $49 \%$ durante los primeros 30 minutos y de más del $72 \%$ durante la primera hora (12).

\section{Transmisión e interpretación de imágenes}

Se identificaron 15 estudios que reportaron el medio de transmisión de las imágenes a las centrales de telemedicina (Ver tabla 3 ), de los cuales 12 solo usaron un sistema y/o aplicación web para la transferencia, informando a las centrales vía telefónica $(2,6,7,10,12,15-21)$. Reponen et. Al, en su estudio piloto, hacían la transferencia a través de mensajes multimedia desde sus teléfonos y un aplicativo web conectado a internet (13), por otro lado, Yamada et. Al, enviaban las imágenes a través de correo electrónico desde sus teléfonos y eran abiertas desde los computadores portátiles ubicados en la central (14). Alias et. Al en su estudio observacional prospectivo no aleatorizado establecieron el papel de telemedicina comparando el uso de mensajes multimedia desde sus teléfonos, de correo electrónico y la transferencia usando un aplicativo web. Mediante la prueba de chi-cuadrado, los tres modos de sistemas de transferencia de imágenes fueron comparados: $(P=0,424$, aplicativo web frente a correo electrónico), $(P=0,324$, correo electrónico frente a mensaje multimedia desde el teléfono) y $(P=0,169$, aplicativo web frente a mensaje de teléfono multimedia). Los resultados indicaron claramente que no hubo diferencia entre las modalidades que compararon de transmisión de imágenes que compararon (11).

Se identificaron 8 estudios en los que se informó qué profesionales estaban a cargo de la interpretación de las imágenes (Ver tabla 4). En los estudios de Reponen et. Al, Pagani et. Al y Erly et. Al, la imagen fue recibida por un radiólogo inicialmente, haciendo una interpretación preliminar seguido por la valoración de neurorradiólogos para interpretación final $(7,13,15)$. Poca et. Al en su estudio, se valieron de neurocirujanos y neurorradiólogos para la interpretación de las imágenes transferidas (21). Las imágenes en el ensayo clínico de Wong et. A, fueron interpretadas por neurocirujanos, quienes decidían si la transferencia había sido o no innecesaria; el tiempo del proceso de consulta para el grupo de videoconferencia $(1,30 \pm 2,5$ h) fue mayor en comparación con la teleconsulta $(0,70 \pm 1,9 h ; P=0,003)$, pero comparable a la telerradiología $(1,01 \pm 1,8 \mathrm{~h} ; \mathrm{P}=0,147)$ (10). Ashkenazi et. Al y Mrak et. Al, usaron radiólogos para la interpretación inicial, el cual consultaba con neurocirujanos en caso de hallazgos patológicos $(16,22)$. Por último, en el estudio de Franczak et. Al, la interpretación estuvo a cargo de tres neurorradiólogos con experiencia en imágenes clínicas (23). 
Tabla 3 Transmisión de imágenes

\begin{tabular}{|c|c|c|c|c|}
\hline Autores & mensaje multimedia & correo electrónico & Sistema/ interface web & Referencia \\
\hline Goh et al (1997) & & & & (17) \\
\hline Goh et al (1997) & & & & (2) \\
\hline Reponen et al (2000) & & & & (13) \\
\hline Pagani et al (2003) & & & & (15) \\
\hline Erly et al (2003) & & & & (7) \\
\hline Yamada et al (2003) & & & & (14) \\
\hline Yaghmai et al (2003) & & & & (19) \\
\hline Poca et al (2004) & & & & (21) \\
\hline Wong et al (2006) & & & & (10) \\
\hline Zulu et al (2007) & & & & (6) \\
\hline Fabbri et al (2008) & & & & (20) \\
\hline Kreutzer et al (2008) & & & & (18) \\
\hline Mrak et al (2009) & & & & (16) \\
\hline Alías et al (2013) & & & & (11) \\
\hline Dario et al (2014) & & & & (12) \\
\hline
\end{tabular}

Tabla 4. Encargados de la interpretación de las imágenes

\begin{tabular}{llll}
\hline Autores & Radiólogo & Neurocirujano & Neurorradiólogo \\
\hline Reponen et al (13) & & \\
Pagani et al (15) & \\
Erly et al (7) & \\
Poca et al (21) & \\
Wong et al (10) & \\
Ashkenazi et al (22) \\
Mrak et al (16) \\
Franczak et al (23)
\end{tabular}

\section{Costos de la telerradiología}

Cuatro estudios mencionaron factores relacionados con el costo de la telerradiología, el trabajo de Reponen et. Al, solo especificó el costo de la implementación de la tecnología PDA utilizada para la transmisión de imágenes, con un valor aproximado de mil euros (13). Por otro lado, en el estudio de Kreutzer et. Al, ejecutado en 7 hospitales alemanes durante un periodo de 5 años, el valor de la implementación del sistema de transferencia "CP220" fue de 12 mil euros en cada hospital, para un total de 96 mil euros entre el grupo de hospitales involucrados en el estudio, sin embargo, este es un valor referencial de lo reportado por los autores. Adicionalmente, este mismo estudio determinó el costo del transporte terrestre del equipo médico y del paciente, según la severidad de las lesiones, con un rango de precios que oscilan entre los 508 y los 559 euros (18). Otro estudio realizado en Japón especificó que el valor monetario de la transmisión de las imágenes fue de 0,18 dólares, usando el J-Phone SHO7 (24). Mientras que Wong et. Al, en su 
estudio llevado a cabo en Hong Kong, mencionó un valor de 33,016 dólares como costo de la consulta por telerradiología usando un software de Windows (25). No fue posible estimar un valor promedio utilizando los diferentes tipos de medios usados para la telerradiología, por tanto, los anteriores valores corresponden solo a lo reportado por los autores de los manuscritos.

Examen imagenológico enviado por telerradiología y diagnósticos reportados

Dentro de los 21 estudios revisados, 20 $(95,23 \%)$ reportaron el examen imagenológico usado y enviado por telerradiología. De estos, el examen más solicitado fue la tomografía computarizada (TC) con 15 artículos que representa el $68,18 \%$ de las muestras. Seguido por 2 artículos $(9,52 \%)$ que tenían la solicitud conjunta de TC y resonancia magnética (RM), que fueron el estudio de Kreutzer et. Al, en donde se usó un sistema de transferencia de imágenes realizadas por TC y RM en 7 hospitales en el sur de Alemania (18); y el estudio de Wai Hoe $\mathrm{Ng}$ et. Al, donde también usaron TC y RM enviados a través de mensajes por celular (1). Dos Estudios (9,52\%), Latifi et. Al y Alias et. Al, no especificaron el tipo de examen imagenológico usado durante la telerradiología $(11,26)$. Dario et al, mencionaron el uso del TC y/o RM representando un $(4,5 \%)$ de la muestra (12). Así mismo un solo estudio realizado por Goran Mark et al, empleó tanto TC como RM y además angiografía por sustracción digital (DSA) (16), diferenciándose de un estudio hecho por Yamada et. Al, quienes también usaron los tres métodos imagenológicos, sin embargo, la angiografía fue convencional, es decir, sin sustracción digital (14).

En cuanto a los diagnósticos emitidos la mayoría fueron en torno a trauma cerebral; el estudio de Poca et. Al, dividió el diagnóstico de trauma en leve, moderado y severo y a su vez si la lesión era difusa en (tipo I, II, III, IV) o focal (evacuadas o no evacuadas) (21). División parecida a la realizada en el estudio de Alias et. Al, quienes también lo organizaron según la gravedad en leve, moderado y severo (11). Por otro lado, en el estudio de Fabbri et. Al, se pudo demostrar que el hematoma subdural era el diagnóstico más frecuente secundario al trauma, seguido del hematoma intracerebral, la fractura basal de cráneo y el hematoma epidural (20). En cuanto al mecanismo del trauma Zulu et. Al, describieron que el trauma cerrado fue la forma más frecuente, seguido del trauma por arma de fuego y el trauma por arma blanca (6).

\section{Discrepancias en el diagnóstico y fallas del sistema}

De los artículos revisados se determinó en 6 de ellos algún tipo de falla en el sistema o discrepancias en los conceptos emitidos $(2,7,13,15,17,21)$. En el estudio de Goh, Lam, \& Poon, de los dos grupos estudiados en términos de precisión diagnóstica, hubo un buen acuerdo de diagnóstico, con solo tres casos diagnosticados erróneamente en el grupo dos. Un caso de hemorragia subaracnoidea diagnosticada como encefalitis viral, un tumor ventricular, diagnosticado como hemorragia intraventricular, y un tercer caso de hemorragia intraventricular en el tercer y cuarto ventrículos, diagnosticada como un hematoma cerebeloso (2). Para ese año también Goh, Tsang, \& Poon, mostraron solo un caso de presencia de hemorragia subaracnoidea de origen traumático omitida por el médico remitente, pero sin ningún impacto en el manejo del paciente en la fase aguda (17). Reponen et. Al, reportaron en 3 casos en que la conexión de datos se perdió antes que todas las imágenes fueran transferidas (13). Luego Pagani et. Al, encontraron que algunos hallazgos radiológicos pasaron desapercibidos, entre ellos cinco casos de infarto (un temporoparietal izquierdo, un frontoparietal, dos parietales izquierdos y un infarto interno cápsula derecho) y 3 fracturas óseas reportadas (una fractura de hueso temporal y dos fracturas occipitales óseas izquierdas) (15). En ese mismo año, el estudio de Erly et. Al, encontró que en 39 casos las conclusiones iniciales y finales no estuvieron de acuerdo, de las cuales 16 se consideraron como errores significativos y 23 fueron insignificantes (7).

Posteriormente, Poca et. Al, en 2004, observaron que de las 90 exploraciones valoradas por el neurocirujano de guardia o por el neurorradiólogo, hubo discrepancias en 4 casos de ellos cuando se realizó la valoración de forma diferida. Sin embargo, ninguno presentó complicaciones 
posteriores; asimismo se reportaron inconvenientes técnicos durante los primeros meses del estudio, siendo necesario en ciertos casos la realización de una llamada telefónica para alertar al centro receptor de las dificultades en envío del fax o de las malas condiciones de recepción del informe. Por otro lado, se informó que en los primeros 9 meses existieron problemas de transmisión de las imágenes en 3 pacientes (21).

\section{Mortalidad, fallas de tratamiento, deterioro neurológico y secuelas derivadas}

De los estudios analizados se encontró que en 8 de ellos se reportaron los tópicos de mortalidad, secuelas derivadas, fallas de tratamiento o deterioro neurológico. En el año 1997, Goh et. Al, analizaron dos grupos de pacientes referidos con o sin la colaboración del sistema de telerradiología, encontrando una cifra de mortalidad global del $14 \%$ (17). Por otro lado, en ese mismo estudio se encontró que la incidencia de injuria secundaria durante la transferencia fue del 32\% (9 de 28 pacientes) en el grupo sin uso de telerradiología, de los cuales la hipoxia e hipotensión ocurrió en dos instancias. Mientras que en el grupo con la implementación del sistema, la incidencia de eventos adversos fue sólo del 6,4\% (2 de 31 pacientes), presentándose hipotensión en uno de los dos casos reportados. En general los pacientes que sufrieron injuria secundaria durante la transferencia tuvieron resultados pobres a los seis meses. De esta forma, de los 9 pacientes del grupo sin ayuda por telerradiología, el $44 \%$ quedaron en estados vegetativos o murieron y el porcentaje restante presentó discapacidad moderada o severa. De igual forma, de los 2 pacientes con efectos adversos del grupo con uso de telerradiología, uno murió y otro permaneció en coma (17).

En ese mismo año, en un estudio también de dos grupos documentaron que la incidencia de eventos secundarios durante la transferencia fue del $32 \%$ ( 16 de 50 pacientes) en el grupo 1, los eventos adversos críticos que comprenden el $8 \%$ (4 de 50 individuos) y los eventos graves el $24 \%$ (12 de 50 sujetos). En comparación, la incidencia de injuria en el grupo 2 fue del $8 \%$ ( 4 de 52 ), de estos, $4 \%$ ( 2 de 52 ) fueron eventos críticos y $4 \%$ ( 2 de 52 ), graves. En general, se produjeron eventos hipóxicos en el $6 \%$ ( 6 de 102) de las transferencias. Además, se determinó que el resultado a 1 mes en el grupo 1 fue la muerte en el $44 \%$ ( 7 de 16 ) de los pacientes con injuria secundaria, discapacidad grave en el $25 \%$ (4 de 16) y discapacidad moderada en $31 \%$ (5 de 16 ). En el grupo 2, hubo una muerte (25\%) entre los pacientes con transferencias inseguras, dos $(50 \%$, 2 de 4 ) quedaron severamente discapacitadas y uno $(25 \%)$ moderadamente discapacitado. No hubo muertes durante la transferencia o muertes prematuras en la UCI (2).

Servadei et. Al, informaron en su estudio solo tres muertes entre los pacientes, de 10 de los pacientes que fueron referidos, el primero un aumento de tamaño de un hematoma subdural, que se deterioró y que fue evaluado inicialmente como no quirúrgico; un segundo caso resultado de una ruptura de bazo y un último caso de deterioro clínico de un paciente con múltiples contusiones (27).

Ashkenazi et. Al, encontraron en uno de los grupos de sus pacientes, los cuales estaban hospitalizados en un centro de trauma de nivel 2 , donde hubo dos pacientes $(2,8 \%)$ a quienes definieron como fallas de tratamiento, uno de ellos falleció (22). Por otra parte, en el estudio de Zulu et. Al, 265 pacientes fueron manejados en la unidad de cirugía general (GSU) y 51 fueron transferidos a la unidad de neurocirugía (NSU). De este último grupo, dos muertes se dieron durante el traslado a la NSU y una a la llegada a la NSU. Cuarenta y dos de los 48 restantes fueron sometidos a varios procedimientos neuroquirúrgicos y 6 pacientes fueron admitidos en la NSU para observación neuroquirúrgica. Ocho pacientes murieron después de una cirugía cerebral. En total, 41 pacientes fallecieron (tasa de mortalidad del $13 \%$ ), además de los 42 pacientes en quienes se informó un Glasgow de 8 , cerca del $66 \%$ fallecieron, en comparación al $4,7 \%$ de los pacientes que fallecieron con un Glasgow $>8$ ( $p<0,0001)$. Hubo 11 pacientes con graves secuelas en la GSU, que comprendían hemiparesias, monoparesias y 12 pacientes con secuelas graves en la NSU: hemiplejia, hemiparesia y monoparesia (6).

En 2008 Kreutzer et. Al, reportaron en su estudio una tasa de mortalidad del $4 \%$ (18). Du- 
rante ese mismo año, Fabbri et. Al, evidenciaron un resultado desfavorable en 90 casos $(10,5 \%)$ de 865 participantes, 24 pacientes murieron: 12 durante el período de hospitalización, 12 durante el seguimiento de 6 meses, 5 de los pacientes se declararon en estado vegetativo permanente y 61 quedaron en condición de discapacidad severa (20).

Finalmente, Ashkenazi et. Al en 2015, evidenciaron que de los 48 ( $8,5 \%$ del total) pacientes transferidos tardíamente al centro de trauma de primer nivel 1,24 pacientes murieron. De estos, la mitad de los pacientes murieron al poco tiempo de llegar al servicio de emergencias, tres de ellos fueron estabilizados después de la reanimación o cirugía y la evaluación neuroquirúrgica después de la estabilización reveló la pérdida completa de la función cerebral y estos pacientes finalmente murieron. Luego de varios días de hospitalización, 2 pacientes murieron de lesiones asociadas $y$ otros 2 murieron por complicaciones de sus comorbilidades. De este grupo de 48 pacientes se investigó las fallas en la intención de tratar, de las cuales solamente 23 fueron encontradas como fallas reales (28).

\section{DISCUSIÓN}

Dentro de los hallazgos de esta revisión, se encontró que los estudios en su mayoría utilizaron como medio para realizar la telerradiología las líneas telefónicas acompañada de otros medios como la mensajería multimedia; datos que son similares con los reportados por otros autores, donde demostraron que el uso de mensajería multimedia mediante un teléfono móvil, era el medio preferido para realizar la telerradiología; y que este sistema, aumentó significativamente el nivel de confianza a la hora de tomar decisiones sólidas iniciales a diferencia de nuestros resultados, donde 6 estudios, reportaron fallas en el sistema y discrepancia en el diagnóstico por imagen e incluso uno de los estudios informó una diferencia significativa en 16 casos entre las conclusiones iniciales y finales al momento de interpretar las imágenes $(21,29)$.

Otros autores refieren que quedan varias cosas por elaborar antes que el teléfono celular se utilice como terminal formal en telerradiología, ya que los datos inalámbricos, el proceso de las imágenes, la resolución de la pantalla y la sensibilidad al tacto, se postulan como impedimento para esto $(25,26)$; en concordancia con ello, uno de los estudios incluidos en este manuscrito reveló que en 3 ocasiones las imágenes no pudieron cargar, debido a que quedaron sin conexión de datos (13). Por tanto, en situaciones de emergencia la velocidad de los MMS es mucho más relevante. Asimismo, la TC cerebral ha sido utilizada de manera frecuente en casos de emergencias neurológicas; este hallazgo respalda los resultados de esta revisión, donde se reportó que, en 15 de los estudios incluidos el examen más solicitado fue la TC (13).

Es importante destacar que la telerradiología no es solo el hecho de compartir imágenes a través de aplicaciones o dispositivos móviles, pues se trata más de una relación a distancia entre especialistas médicos con mediación de conocimiento que facilita el acceso a informes radiológicos que apoyan el diagnóstico de las patologías, en este caso en particular, del neurotrauma, especialmente en situaciones donde la distancia geográfica o la falta de servicios especializados juega un rol fundamental en el tratamiento y pronóstico de los pacientes (30). Pese a que muchos manuscritos abordaron la implementación de la telerradiología, incluso los costos, no fue posible determinar ubicaciones geográficas o especificar las limitaciones de acceso que tenían los centros donde se llevaron a cabo estas investigaciones, ya que no se contaba con descripciones detalladas a este nivel. Otra limitante fue que en cuanto al costo de la tecnología no fue posible determinar el valor aproximado, ya que la mayoría de los textos mencionaba el valor de la implementación en el servicio como tal, pero no se especificaba el gasto mensual promedio de cada tipo de tecnología.

Esta revisión abre la puerta a futuros trabajos donde se puedan explorar tanto el costo como la determinación de las ventajas sobre el uso de esta tecnología en neurotrauma, especialmente en lugares donde las condiciones de acceso se dificultan por la ubicación geográfica de los centros de salud a nivel mundial, incluso teniendo en cuenta las restricciones de la actualidad como 
las que aparecen ante una situación de pandemia como la que se vive hoy en día.

\section{CONCLUSIONES}

La implementación de nuevas estrategias como la telerradiología pueden ofrecer a zonas de difícil acceso y/o carentes de neurocirujanos presenciales la posibilidad de evaluaciones oportunas para definir manejos de patologías traumáticas. El diseño de protocolos de manejos adaptados a países o zonas austeras serán requeridas para garantizar el éxito de la tele radiología en neurotrauma.

Estudios como el actual demuestran que el uso de la telerradiología puede reducir el traslado innecesario de pacientes a centros de mayor complejidad; lo cual impacta de manera positiva los costos en atención en salud.

\section{REFERENCIAS BIBLIOGRÁFICAS}

1. Ng WH, Wang E, Ng I. Multimedia Messaging Service teleradiology in the provision of emergency neurosurgery services. Surg Neurol. 2007;67(4):338-41.

2. Goh KY, Lam CK, Poon WS. The impact of teleradiology on the inter-hospital transfer of nenrosurgical patients. $\mathrm{Br}$ J Neurosurg. 1997;11(June 1996):52-6.

3. Bradley WG. Teleradiology. Neuroimaging Clin N Am. 2012;22(3):511-7.

4. Chen L, Mao Y. The value of teleradiology in neurosurgery. World Neurosurg. 2012;77(2):285-6.

5. Gosselin RA, Spiegel DA, Coughlin R, Zirkle LG. Injuries: the neglected burden in developing countries. Bull World Health Organ. 2009;87(4):246-246a.

6. Zulu BMW, Mulaudzi T V, Madiba TE, Muckart DJJ. Outcome of head injuries in general surgical units with an off-site neurosurgical service. Injury. 2007 May; 38(5):576-83.

7. Erly WK, Ashdown BC, Lucio RW 2nd, Carmody RF, Seeger JF, Alcala JN. Evaluation of emergency CT scans of the head: is there a community standard? AJR Am J Roentgenol. 2003 Jun;180(6):1727-30.

8. Fabbri A, Servadei F, Marchesini G, Stein SC, Vandelli A. Observational approach to subjects with mild-tomoderate head injury and initial non-neurosurgical lesions. J Neurol Neurosurg Psychiatry [Internet]. 2008;79(10):1180-5. Disponible en: http://www. embase. $\mathrm{com} / \mathrm{search} /$ results? subaction = viewrecord\&from $=$ export\&id $=\mathrm{L} 352451888$
9. Ashkenazi I, Zeina A., Kessel B, Peleg K, Givon A, Khashan $\mathrm{T}$, et al. Effect of teleradiology upon pattern of transfer of head injured patients from a rural general hospital to a neurosurgical referral centre: follow-up study. Emerg Med J. 2015 Dec 1;32(12):946-50.

10. Wong HT, Poon WS, Jacobs P, Goh KYC, Leung CHS, Lau $\mathrm{FL}$, et al. The comparative impact of video consultation on emergency neurosurgical referrals. Neurosurgery. 2006;59(3):607-12.

11. Alias A, Remeli AZ, Mohammed Haspani MS. Teleconsultation in neurosurgery: Comparing the multimodal approach in image transfer in Kuala Lumpur hospital. Malaysian J Med Sci. 2013;20(1):95-6.

12. Dario C, Scannapieco G, Scienza R, Carraro MG, Saccavini C, Vio $E$, et al. The neurosurgical telecounseling network in the Veneto Region: 4 years of experience of HEALTH OPTIMUM. Telemed J E Health [Internet]. 2014 Nov;20(11):1009-14. Disponible en: http://www.embase.com/search/results?subaction $=$ viewrecord\&from $=$ export\&id $=\mathrm{L} 612370471$

13. Reponen J, Ilkko E, Jyrkinen L, Tervonen O, Niinimäki J, Karhula $\mathrm{V}$, et al. Initial experience with a wireless personal digital assistant as a teleradiology terminal for reporting emergency computerized tomography scans. J Telemed Telecare [Internet]. 2000;6(1):45-9. Disponible en: http://www.embase.com/search/results?subaction $=$ viewrecord\&from $=$ export\&id $=\mathrm{L} 31326377$

14. Yamada $M$, Watarai $H$, Andou T, Sakai $N$, Martín-Rodríguez JG, MacDonald JD, et al. Emergency image transfer system through a mobile telephone in Japan: Technical note. Neurosurgery. 2003;52(4):986-90.

15. Pagani L, Jyrkinen L, Niinimäki J, Reponen J, Karttunen A, Ilkko E, et al. A portable diagnostic workstation based on a Webpad: implementation and evaluation. J Telemed Telecare [Internet]. 2003;9(4):225-9. Disponible en: http://www.embase.com/search/results? subaction =viewrecord\&from $=$ export\&id $=\mathrm{L} 137557578$

16. Mrak G, Paladino J, Dzubur A, Desnica A. Telemedicine in neurosurgery-Teleradiology connections in the Republic of Croatia. J Telemed Telecare. 2009;15(3):142-4.

17. Goh KY, Tsang KY, Poon WS. Does teleradiology improve inter-hospital management of head-injury? Can J Neurol Sci. 1997 Aug;24(3):235-9.

18. Kreutzer J, Akutsu H, Fahlbusch R, Buchfelder M, Nimsky $C$, J. K, et al. Teleradiology in neurosurgery: experience in 1024 cases. J Telemed Telecare [Internet]. 2008;14(2):67-70. Disponible en: http://www.embase. $\mathrm{com} / \mathrm{search} /$ results? subaction $=$ viewrecord\&from $=\mathrm{ex}-$ port\&id $=\mathrm{L} 352140303$

19. Yaghmai V, Salehi SA, Kuppuswami S, Berlin JW. Rapid wireless transmission of head CT images to a personal digital assistant for remote consultation. Acad Radiol. 2004 Nov;11(11):1291-3.

20. Fabbri A, Servadei F, Marchesini G, Stein SC, Vandelli A. Observational approach to subjects with mild-tomoderate head injury and initial non-neurosurgical lesions. J Neurol Neurosurg Psychiatry [Internet]. 2008;79(10):1180-5. Disponible en: https://id.elsevier.com/as/authorization.oauth2?platSite $=\mathrm{SC} \% 2 \mathrm{Fscopus} \& u i$ locales $=$ en-US\&scope $=$ openi- 
$\mathrm{d}+$ profile+email+els auth info+els analytics info +urn\%3Acom\%3Aelsevier\%3Aidp\%3Apolicy\%3Aproduct\%3Aindv identity\&response type $=$ code\&redirect uri=https $\% 3 \mathrm{~A} \% 2 \mathrm{~F} \% 2 \mathrm{Fwww}$. scopus.com\%2Fauthredirect. uri\%3FtxGid\%3Dc5daa9b1a3c9ae835f58f638628ec232\&state=forceLogin\%7CtxId\%3D0C09ADCE4E4F610465690790EF2E5630.i-0ad5396539706f2c4\%3A5\&authType=SINGLE SIGN IN\&prompt=login\&client id $=$ SCOPUS

21. Poca MA, Sahuquillo J, Domenech P, Pedraza S, Maideu J, Vila $X$, et al. [Use of teleradiology in the evaluation and management of head-injured patients. Results of a pilot study of a link between a district general hospital and a neurosurgical referral center]. Neurocirugia (Astur). 2004 Feb;15(1):17-35.

22. Ashkenazi I, Zeina A., Kessel B, Peleg K, Givon A, Khashan $T$, et al. Effect of teleradiology upon pattern of transfer of head injured patients from a rural general hospital to a neurosurgical referral centre. Emerg Med J [Internet]. 2007 Aug;24(8):550-2. Disponible en: http://www.embase.com/search/results? subaction=viewrecord\&from $=$ export\&id $=\llcorner 47193723$

23. Franczak MJ, Klein M, Raslau F, Bergholte J, Mark LP, Ulmer JL. In emergency departments, radiologists' access to EHRs may influence interpretations and medical management. Health Aff (Millwood). 2014 May;33(5):800-6.

24. Yamada M, Watarai H, Andou T, Sakai N. Emergency image transfer system through a mobile telephone in Japan: technical note. Neurosurgery. 2003 Apr;52(4):986-90.

25. Poon WS, Wong H, Goh KY., Kwok SP., Jacobs P. The comparative impact of videoconsultation on emergen- cy neurosurgical referrals. Hong Kong Med J [Internet]. 2007;13(5):13-4. Disponible en: http://www.embase. $\mathrm{com} /$ search/results? subaction $=$ viewrecord\&from $=$ export\&id $=\mathrm{L} 610342935$

26. Latifi R, Gunn JKL, Bakiu E, Boci A, Dasho E, Olldashi $F$, et al. Access to Specialized Care Through Telemedicine in Limited-Resource Country: Initial 1,065 Teleconsultations in Albania. Telemed J E Health [Internet]. 2016 Dec;22(12):1024-31. Disponible en: http://www. embase. $\mathrm{com} / \mathrm{search} /$ results? subaction = viewrecord\&from $=$ export\&id $=\mathrm{L} 619702093$

27. Servadei F, Antonelli V, Mastrilli A, Cultrera F, Giuffrida $M$, Staffa $G$. Integration of image transmission into a protocol for head injury management: a preliminary report. Br J Neurosurg. 2002;16(1):36-42.

28. Ashkenazi I, Zeina A., Kessel B, Peleg K, Givon A, Khashan $\mathrm{T}$, et al. Effect of teleradiology upon pattern of transfer of head injured patients from a rural general hospital to a neurosurgical referral centre: Follow-up study. Emerg Med J [Internet]. 2015;32(12):946-50. Disponible en: http://www.embase.com/search/results? subaction $=$ viewrecord\&from $=$ export\&id $=\mathrm{L} 607210743$

29. Latifi R, Olldashi F, Dogjani A, Dasho E, Boci A, El- Menyar A. Telemedicine for Neurotrauma in Albania: Initial Results from Case Series of 146 Patients. World Neurosurg [Internet]. 2018;112:e747-53. Disponible en: http://www.sciencedirect.com/science/article/pii/ S187887501830189X.

30. Martí-Bonmatí L, Morales A, Donoso L. Hacia un uso adecuado de la telerradiología. Radiología. 2012; 54(2): 115-123. 


\section{Fuente de financiación:}

Recursos propios de los autores.

\section{Conflictos de intereses:}

Los autores declaran no tener conflictos de intereses.

Autor para correspondencia:

Yelson Alejandro Picón-Jaimes.

ypicon@unab.edu.co

Editor:

Prof. Dr. Felipe Villela Gomes

Received in: jul 30, 2020

Approved in: dec 18,2020

(c) (i) Este é um artigo publicado em acesso aberto (Open Access) sob a licença Creative 